\title{
The Comparison of the Manifestation of the Clinical Imageology and Pathology between the Brucellar Spondylitis and the Spine Turberculosis*
}

\author{
Xinming Yang ${ }^{1 \#}$, Xianyong Meng ${ }^{1}$, Wei Shi' ${ }^{2}$, Yakun Du ${ }^{3}$, Lei Zhang ${ }^{4}$, Yaoyi Wang ${ }^{1}$ \\ ${ }^{1}$ Department of Orthopaedics, The First Affiliated Hospital of Hebei North University, Zhangjiakou, China \\ ${ }^{2}$ Department of Paediatrics, The First Affiliated Hospital of Hebei North University, Zhangjiakou, China \\ ${ }^{3}$ Department of Neurology, The Children’s Hospital of Hebei Province, Shijiazhuang, China \\ ${ }^{4}$ Department of Neurosurgery, The Fourth Affiliated Hospital of Hebei Medical University, Shijiazhuang, China \\ Email: ${ }^{\text {yxm1120@sohu.com }}$
}

Received November 27, 2013; revised December 25, 2013; accepted January 3, 2014

Copyright (c) 2014 Xinming Yang et al. This is an open access article distributed under the Creative Commons Attribution License, which permits unrestricted use, distribution, and reproduction in any medium, provided the original work is properly cited. In accordance of the Creative Commons Attribution License all Copyrights ( 2014 are reserved for SCIRP and the owner of the intellectual property Xinming Yang et al. All Copyright (C) 2014 are guarded by law and by SCIRP as a guardian.

\section{ABSTRACT}

Objective: To improve the clinical differential diagnosis level, the clinical manifestation of the brucellar spondylitis and the spine turberculosis were discussed in this paper. Method: The study was completed in the No. 1 Affiliated Hospital of Hebei North University in Zhangjiakou City, Hebei Province, China, from January 2001 to December 2013 by Analyzing the X-ray, CT scanning and MRI of 257 cases of the brucellar spondylitis retrospectively and comparing with the clinical imageology and pathology 332 cases of turberculosis of the spine diagnosed finally. Results: The brucellar spondylitis: The focuses usually locate in the lumbar vertebra and L4, 5 has the highest occurrence rate. The focuses are often small but multiple, and limited to the edge of the vertebra. Hyperostosis and osteoscterosis are usually found in the tissuses around the focuses. There are often new focuses in the newborn bones, and the destruction of intervertebral discs is usually slight. Hyperostosis and osteoscterosis might be found in the surfaces of the joints. The densites of the bones close to the focuses become high. There were less or no paravertebral abscesses but inflammational granuloma can be found frequently. Turberculosis of the spine: The focuses are usually located in the thoracic and lumbar vertebra, and are characterized by the destruction of the vertebra and the intervertebral discs, accompanied by the appearance of dead bones. In most cases, paravertebral abscesses and osteoporosis might be found. Conclusions: The specific manifestation of the clinical imageology can help to differentiate the brucelar spondylitis from the turberculosis of the spine.

\section{KEYWORDS}

Brucellosis Spondylitis; Turberculosis of the Spine; Tomography; X-Ray Computed; Magnetic Resonance Imaging; Pathology

\section{Introduction}

Brucellosis is caused by Brucella zoonotic infections allergic disease; the disease is often caused by the invasion of infectious spondylitis of the spine or discitis called brucellosis spondylitis, which is in clinical and imaging

*2013 China Hebei Province key medical disciplines tracking project (GL201321); 2013 China Hebei Province, the provincial major medical research projects (zd2013049).

${ }^{\#}$ Corresponding author. learn in many aspects of spinal tuberculosis similar performance, and easily misdiagnosed [1-6]. In recent years, with the increase of Brucella spondylitis disease incidence, epidemiology familiar with the disease, understanding its clinical and imaging findings of its correct diagnosis is important. The author from January 2000 to December 2013 diagnosed and treated for brucellosis spondylitis 257 cases, 332 cases of tuberculosis of the spine, will analyze the following two clinical imaging and pathology. 


\section{Methods}

\subsection{Design}

Comparison of Brucella spondylitis and spinal tuberculosis clinical, radiological and pathological features of the disease to improve the differential diagnosis of the two levels.

\subsection{Time and Study Site}

Research done in China in 2000-01/2013-12 Zhangjiakou City, Hebei Province, the First Affiliated Hospital of Hebei North University.

\subsection{Adoption Standard}

1) with epidemiological history; 2) have spondylitis clinical manifestations; 3) imaging tests, including X-ray, Xray computer tomography (CT), magnetic resonance imaging (MRI); 4) laboratory tests, including serum tube agglutination test (SAT), brucellosis anti-human Immune globulin test, tuberculosis and other clinical specificity of the antibody serology; 5) pathology or pathologic examination including Polymerase Chain Reaction (PCR) and bacterial culture; 6) meet the diagnostic criteria for Brucellosis Endemic Disease Control Division Ministry of Health issued [7].

\subsection{Elimination Standard}

Immature skeletal or malformations, immunosuppressive drugs, patients with spinal trauma or other diseases spinal surgery, history of other patients or spinal infections currently exists.

\subsection{Subjects}

257 cases of Brucella spondylitis patients, 131 male, female 126 cases, aged 21 to 82 years, mean 46 years. All patients had varying degrees of sheep, cattle, or history of exposure to drinking unpasteurized dairy and eating raw beef sterilization, lamb history, including 135 patients living in endemic areas. Before admission underwent X-ray, CT and MRI, 216 cases done in the same part of the X-ray radiography 1 to 2 years ago and CT scans, clinical and radiological misdiagnosed as tuberculosis of the spine. After admission, 257 cases by the local Center for Disease Control and Prevention, prevention and control of communicable diseases standard serum tube agglutination titers were $>1: 160$, rose bengal plate agglutination test (2+), brucellosis anti-human immunoglobulin test drops of 1:400 above, PCR (+). 68 cases positive for pathogenic examination, 257 cases were confirmed by pathological examination of brucellosis. 332 cases of spinal tuberculosis patients over the past decade in the X-ray and CT, MRI examination findings and the clinical, laboratory, surgery and pathology confirmed 145 cases were male, female 187 cases, aged 17 to 78 years, The average 43-year-old. Of which 291 cases of patients with tuberculosis, gastrointestinal history of tuberculosis or history of exposure to tuberculosis.

These patients were slow onset, fever, fatigue, night sweats, neck and shoulder pain, chest pain or persistent low back pain and lumbosacral pain may be accompanied by pain or numbness in limbs radioactive, muscle spasms, spinal movement is limited and limb multi-joint pain and other symptoms.

\section{Results}

257 cases of Brucella spondylitis: 241 cases of two vertebral involvement, $C_{3} 42$ cases, $C_{4-5} 10$ cases, $C_{6} 73$ cases, $\mathrm{C}_{7} \mathrm{~T}_{1} 1$ cases, $\mathrm{T}_{7-8} 1$ cases, $\mathrm{T}_{8-9} 1$ cases, $\mathrm{T}_{9-10} 1$ cases, $\mathrm{T}_{10-11} 2$ cases, $\mathrm{T}_{11-12} 4$ cases, $\mathrm{T}_{12} \mathrm{~L}_{1} 6$ cases, $\mathrm{L}_{1-2} 6$ cases, $\mathrm{L}_{3-4} 59$ cases, $\mathrm{L}_{4-5} 94$ cases, $\mathrm{L}_{5} \mathrm{~S}_{1} 51$ cases; 16 cases three vertebral involvement, $C_{4-6} 2$ cases, $T_{8-10} 2$ cases, $L_{2-4} 3$ cases, $\mathrm{L}_{3-5} 6$ cases, $\mathrm{L}_{4-5} \mathrm{~S}_{1} 3$ cases; lesion distribution the following lumbar highest incidence rate, followed by $\mathrm{L}_{4-5}$ $36.57 \%$ (94/257), L $\mathrm{L}_{3-4} 22.95 \%$ (59/257), $\mathrm{L}_{5} \mathrm{~S}_{1}$ 19.84\% (51/257), most lesions involving the vertebral body to $\mathrm{L}_{4}$. The group of 192 cases of inflammatory infiltration of vertebral bone destruction and multiple small lesions confined to the edge of the vertebral body lesions around the obvious sclerosis, new bone tissue damage foci formation of another new, 41 cases with vertebral facet joint damage, 42 patients with paraspinal abscess formation, no sequestrum; inflammatory infiltration of the group of 204 cases of intervertebral disc, light damage, but it is easy to form the spinal inflammatory granuloma or abscess, including 152 cases of spinal cord, cauda equina or nerve root compression.

332 cases of spinal tuberculosis: Borderline 302 cases, 30 cases center; 286 cases of 2 vertebral involvement, $\mathrm{C}_{1-2} 1$ cases, $\mathrm{C}_{5-6} 5$ cases, $\mathrm{T}_{7-8} 9$ cases, $\mathrm{T}_{8-9} 10$ cases, $\mathrm{T}_{9-10}$ 8 cases, $\mathrm{T}_{10-11} 13$ cases, $\mathrm{T}_{12} \mathrm{~L}_{1} 101$ cases, $\mathrm{L}_{1-2} 56$ cases, $\mathrm{L}_{2-3} 11$ cases, $\mathrm{L}_{3-4} 8$ cases, $\mathrm{L}_{4-5} 39$ cases, $\mathrm{L}_{5} \mathrm{~S}_{1} 25$ cases; 3 vertebral involvement 46 example, $\mathrm{T}_{7-9} 4$ cases, $\mathrm{T}_{8-10} 5$ cases, $\mathrm{T}_{11-12} \mathrm{~L}_{1} 21$ cases, $\mathrm{L}_{2-4} 6$ cases, $\mathrm{L}_{3-5} 7$ cases, $\mathrm{L}_{4-5} \mathrm{~S}_{1} 3$ cases. Thoracolumbar lesion distribution to the highest incidence rate, followed $\mathrm{T}_{12} \mathrm{~L}_{1} 30.42 \%$ (101/332), $\mathrm{L}_{1-2}$ $16.86 \%$ (56/332), $\mathrm{L}_{4-5} 11.74 \%$ (39/332), lesions involving the vertebral body is the most $\mathrm{L}_{1}$. After the group of 332 cases of vertebral lesions confined to the upper and lower edges, and violations of the intervertebral disc and the adjacent vertebral bodies, intervertebral disc damage, disc space narrowing and sequestrum formation is characterized by common paraspinal abscess and osteoporosis, 286 cases of the formation of the spine convex, with little damage to the vertebral body attachment. 


\section{Discussion}

\subsection{Etiology}

Brucella a zoonotic disease caused by Brucella caused by sexually transmitted diseases, occurs in agricultural and pastoral areas in recent years, the global incidence is rising in some areas severe epidemic, the city significantly higher incidence, there are three main vulnerable populations, namely in agricultural and pastoral areas have a history of contact with sick animals; and laboratory staff in contact with the bacterial culture samples; been drinking unpasteurized dairy or sterilization standards eaten uncooked beef, mutton crowd [3,8-11]. Most vulnerable to the disease affects the spine, its epidemiology has evolved from a primarily career-related disease become a major food-borne diseases [10-14]. The more extensive tuberculosis, spinal tuberculosis mainly by tuberculosis, gastrointestinal tuberculosis or lymphatic system caused by spinal infection reaches the blood circulation.

\subsection{Clinical Manifestations}

Brucellosis spondylitis clinical manifestations: 1) relaxation-type fever, fatigue, night sweats, loss of appetite, anemia; 2) associated with other organ infections, respiratory system and reproductive system infections as much, liver, spleen , swollen lymph nodes; 3) multiple, large migratory muscles and joint pain, $\mathrm{L}_{4-5}$ involvement of the most common; 4) persistent low back pain and lower back pain, localized tenderness, percussion pain, with severe neurological roots radiating pain or symptoms of spinal cord compression [10,15-18], muscle cramps, but no kyphosis, less psoas abscess formation, spinal limited mobility, often in a fixed position, has rarely epidural abscess caused paraplegia; 5) Laboratory tests: pathogen isolation, tube agglutination test, complement fixation test, brucellosis anti-human immunoglobulin test positive [4,10-12]; 6) surgical biopsy: noncaseous granulomas $[8,11,17-20]$.

Clinical manifestations of tuberculosis of the spine: 1) afternoon fever, fatigue, night sweats, loss of appetite, anemia; 2) accompanied by other organs of active or old tuberculosis, tuberculosis in the lungs and kidneys as much; 3) to the thoracic spine The most common lumbar $\mathrm{T}_{12} \mathrm{~L}_{1}$ involvement; 4) persistent low back pain and chest and back pain, local percussion pain, radiating pain associated with nerve root and the corresponding muscle spasms, mostly formed paraspinal or psoas abscess, kyphosis, sports disorder, often due to epidural abscess, sequestrum, cheese substance, tuberculosis granulation tissue necrosis caused by spinal disc compression and paraplegia; 5) laboratory tests: pathogen isolation, tuberculin skin test, tuberculosis antibodies [1,4,6,21]; 6) surgical biopsy: caseous necrosis [6,21].

\subsection{Imaging Findings}

Brucella and Mycobacterium tuberculosis infection and can invade any part of the spine [1,3,12,22], the former mostly in the lumbar spine, where the highest incidence of $\mathrm{L}_{4-5}$, the latter thoracolumbar majority, with the highest incidence $\mathrm{T}_{12} \mathrm{~L}_{1}$.

$\mathrm{X}$-ray findings: 1) vertebral inflammation: Brucellosis spondylitis group of 257 cases of bone destruction display borderline lesions showed multifocal, multi against the upper edge of 1 to 2 vertebrae, a minority (16 cases) of three vertebrae. Early performance of small foci of bone thinning, bone defects occur several weeks after the lesions, larger lesions was "islands" shape. Lesion edge sharp, irregular worm-eaten destruction or "knife saw"like appearance (Figure 1), no sequestrum. Of which 48 cases of vertebral centers may have been violated, the center of the vertebral body lesions usually rapid hardening, do not form a deep bone destruction defect, then gradually replaced by new bone, no signs of vertebral compression, do not form kyphosis [23]. TB group showed either center or edge-type type of bone destruction and soon invaded the adjacent disc 2 to 3 vertebrae, there may be jumping destruction, bone destruction was osteolytic lesions, vertebral body may have a hollow cen-

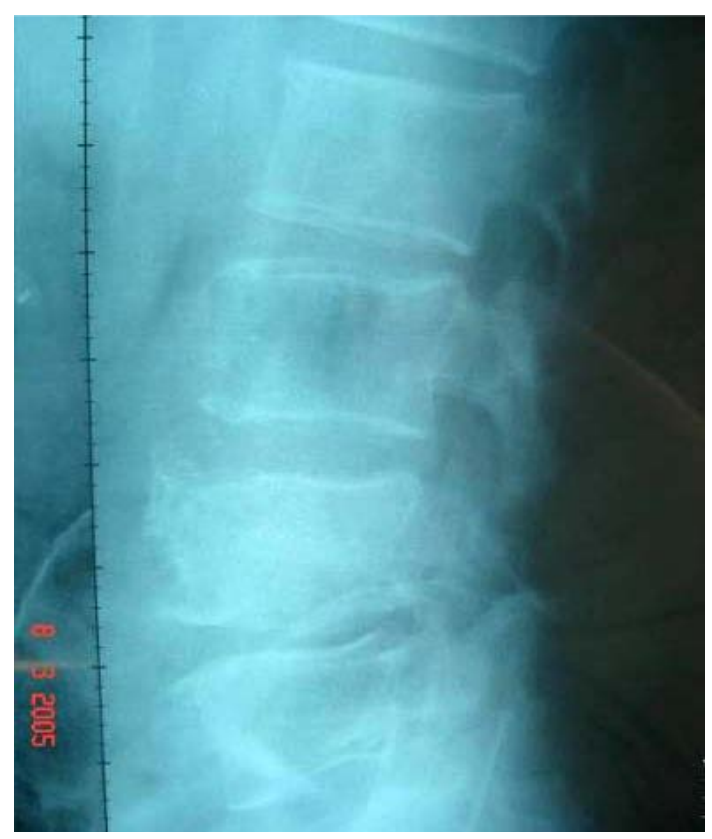

Figure 1. Female, 40 years old, Brucellar spondylitis from the L-3 to L-5. X-ray manifestations: marginal osteoclasia is mulifocality and looks like irregular worm-eaten changes or is an appearance like a knife or saw. The margine of the focus is clear and sharp and the density of the focus is the same as the density of soft tissue. The intervertebral space become narrow, the density become high and the surfaces of the superior and down inferior vertebral bodies become irregular, the tendency of bone destruction combined without dead bones. 
ter, the density is not uniform, and sequestrum formation, widened paraspinal soft tissue, normal muscle shadow blur, chronic cases seen a lot of calcification shadows; late vertebral compression into a wedge, the former narrow width, kyphosis. 2) Discitis: Brucella spondylitis group showed an early disc space narrowing, the density increased, but no damage to the vertebral endplates tendency (Figure 2); tuberculosis group showed progressive destruction of vertebral end plates and intervertebral discs density to reduce uneven intervertebral extremely narrow or disappear (Figure 3).

CT findings: 1) bone changes: Brucella spondylitis group showed multiple lesions of bone destruction no more than $5 \mathrm{~mm}$ in diameter with multiple, round hypodense, coarse trabecular bone disorder, the structure is unclear, edge damage foci obviously with varying degrees of sclerosis, are located in the edge of the vertebral body, new bone See also the new damage foci (Figure 4), a few found in the center of the vertebral body, facet joints also see similar changes (Figure 5), adjacent vertebral density generally increased, no sequestrum and pedicle destruction (Figure 6); spinal tuberculosis as osteolytic bone destruction, destruction of lesion or most often involving the vertebral one. See the destruction of multiple foci of irregular sequestrum, often involving the destruction of the vertebral pedicle rear (Figure 7), adjacent vertebral density generally reduced. 2) changes in the intervertebral disc: both are associated with destruc-

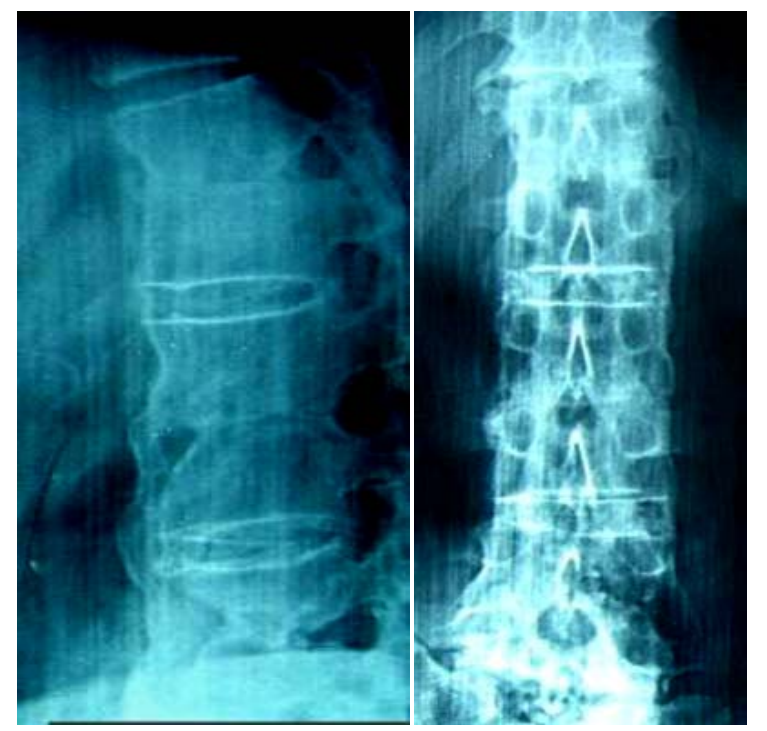

Figure 2. Male, 45 years old, Brucellar spondylitis of the lumbar vertebrae. $X$-ray manifestations:the margins of the verbebral bodies become sclerified and hyperplastic and have formed bone spurs later like a beak which expand to the outside or the adjacent vertebral bodies and the ossification of the anterior and posterior longituinal ligaments formed bone bridges. The disappearance of the intervertebral space and the space of the small joints led to the secondary proliferative arthritis and the the bony ankylosis.

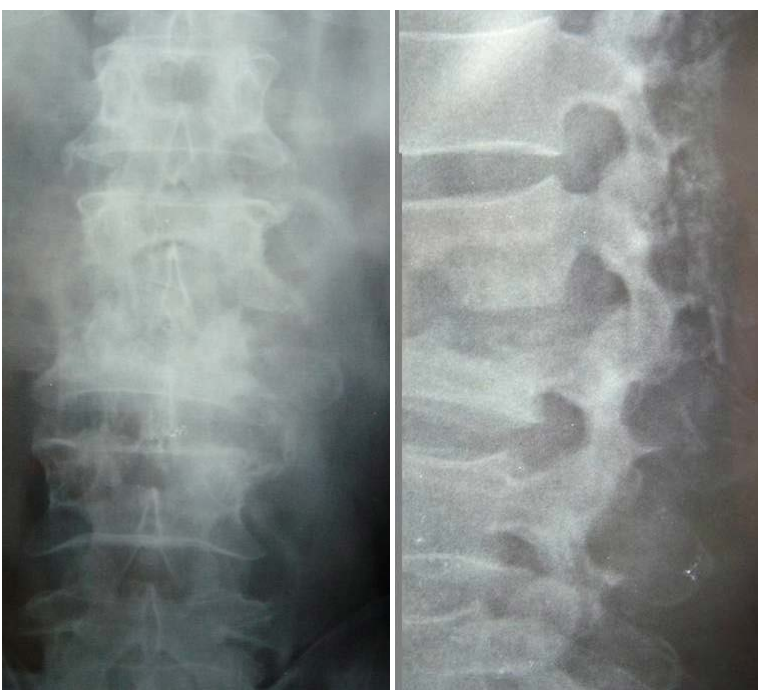

Figure 3. Male, 38 years old, the tuberculosis of the spine from L-2 to L-3. X-ray manifestations: the marginal bone destruction of the vertebral bodies invaded the intervertebral discs and the lamina terminals of the vertebral bodies. The intervertebral space become narrow and the bone destruction is an osteolytic lesion and the cavitates can be found in the centre of the vertebral body. The density was uneven and dead bones formed, the kyphosis combined.

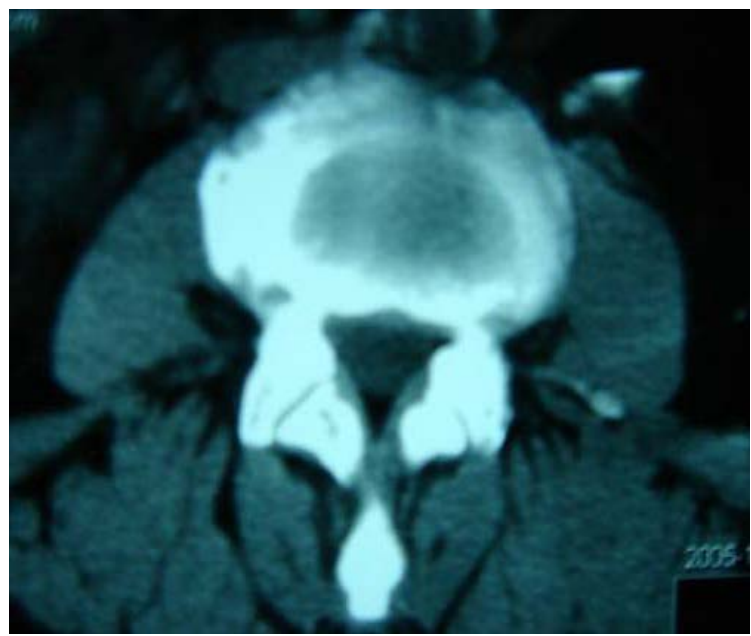

Figure 4. Male, 42 years old, Brucellar spondylitis of the L-4. Manifestation of CT: there were multiple osteoclasia focuses around the vertebral bodies and there were obvious hyperplastic sclerotized strips around the focuses. New destructive focuses can be seen in the newly formed bones.

tion of the adjacent vertebral disc space narrowing, disc damage, but Bruce spondylitis group of CT findings such as bacillary density disc damage is often accompanied by a lot of the same fibrous tissue hyperplasia. As a result, articular surface sclerosis (Figure 8); whereas CT showed tuberculous disc damage, uneven density, there are "stars" like dead bone residue dispersed therein, bone and joint destruction or disappearance of the irregular surface (Figure 9). 3) paraspinal abscess: paraspinal soft 


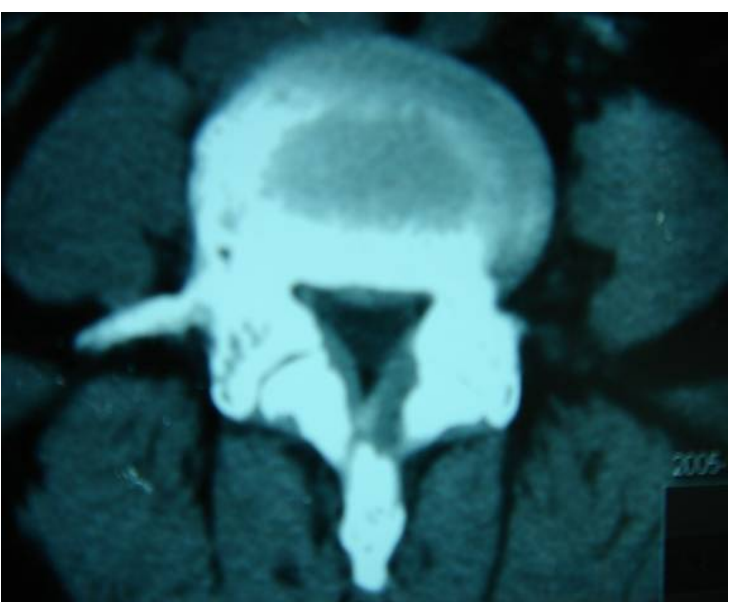

Figure 5. Male, 40 years old, Brucellar spondylitis of the L-4. Manifestation of CT: New destructive focuses can be found in the newly formed bones of the right small joints and the hyperplasia and ossification of the left small joints can also be found.

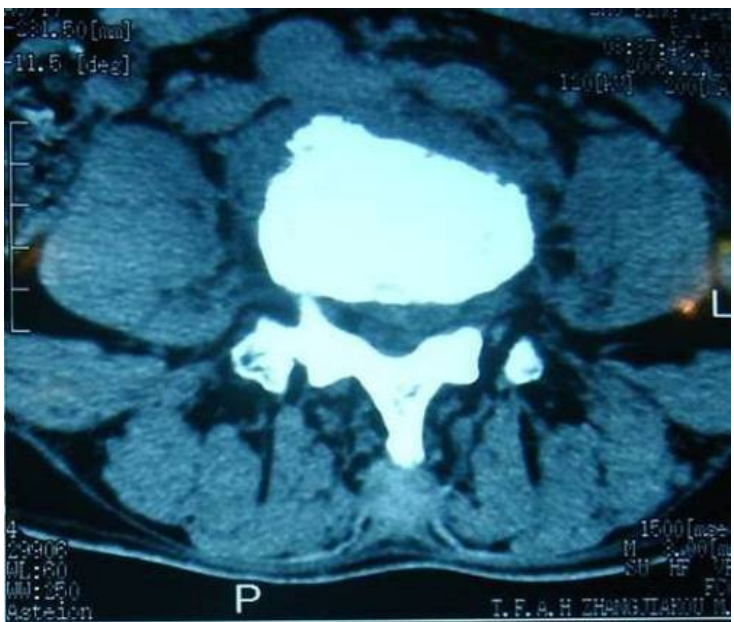

Figure 6. Female, 44 years old, Brucellar spondylitis of the L-4. Manifestation of CT: the density of the adjacent vertebral body of the pathological vertebral body-L-3 become commonly high and the periosteum right border of the vertebral body and the transverse process become hyperplastic and ossific.

tissue between the vertebrae are connected to the damaged area, irregular shape, clear boundaries, pushing the adjacent psoas muscle. Reported in the literature [24-26] Brucella cold abscess disease rarely occurs. The group has 42 cases of vertebral muscle damage plane widened, within which there are limitations abscess formation, clear space around the fat, but there was no immediate signs of flow injection abscess (Figure 10); tuberculosis group not only paraspinal abscess formation, and Common calcification within the abscess, abscess often than vertebra length, forming a single room or multiple rooms or flow injection into the spinal abscess (Figure 11). 4) periosteal changes: Brucella spondylitis group vertebral

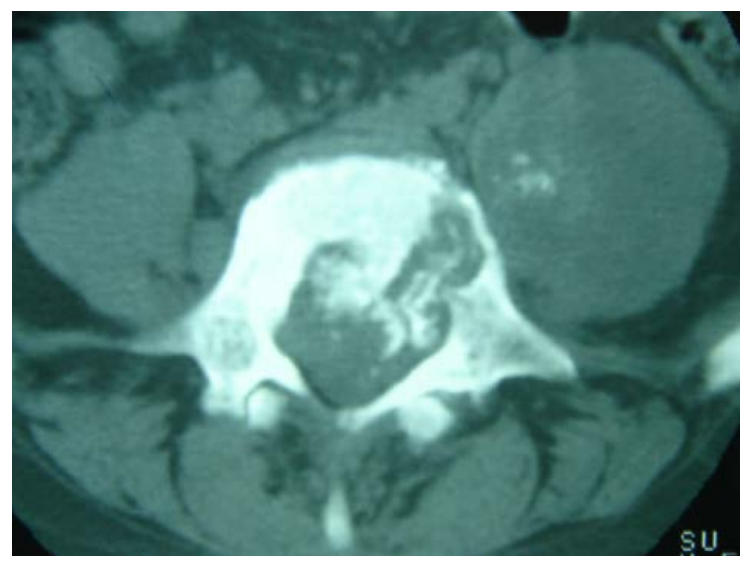

Figure 7. Male, 40 years old, Brucellar spondylitis of the L-4. Manifestation of CT: New destructive focuses can be found in the newly formed bones of the right small joints and the hyperplasia and ossification of the left small joints can also be found.

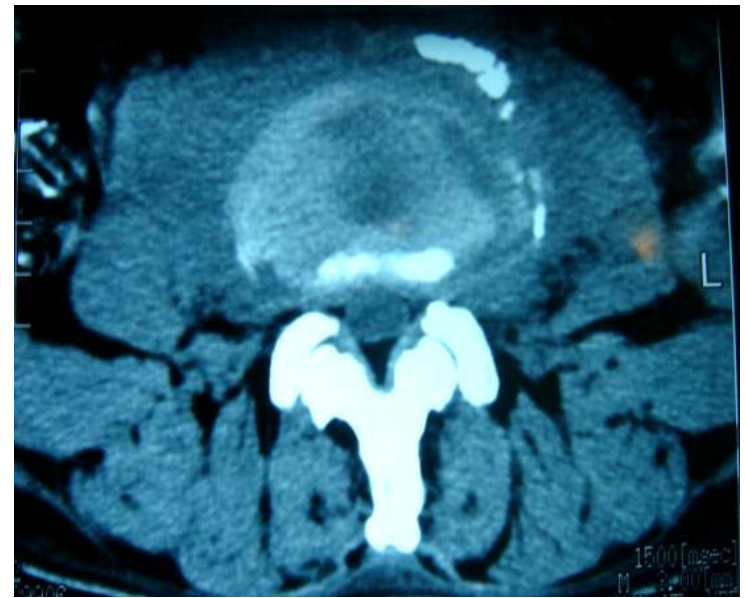

Figure 8. Male, 42 years old, Brucellar spondylitis of the L-4. Manifestation of CT: Destructions of the interverbebral discs and the density of the interverbebral discs was isodensity and the hyperplasy and sclerosis of the articular surfaces can be found. The shadow of soft tissues beside the vertebral bodies was connected to the destructive areas of the vertebral bodies and the shape was irregular and the limit was clear.

periosteal hypertrophy, bulging from the middle to the sides, like the vertebral body mottled uneven density increased, Clostridium deformed vertebra edge periosteal thickening, calcification, form a "lip" like osteophytes (Figure 12), plus the damage foci during freshman osteophytes constitute "lace vertebrae" of the characteristic performance (Figure 13), but between calcification and vertebral periosteum still legible. Adjacent vertebral osteophytes to form fused vertebrae side. Sometimes the performance of the transverse process of the periosteum top hat-shaped transverse thickening. TB group no such performance.

MRI findings: CT features in addition to the two 


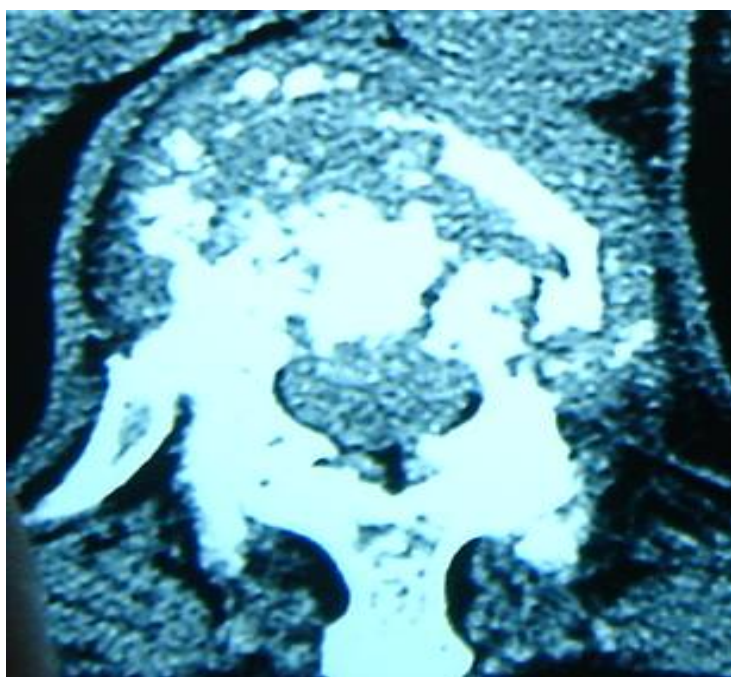

Figure 9. Female, 39 years old, the tuberculosis of the spine of the T-12 and L-1. Manifestation of CT: Destructions of the interverbebral discs and the density of the the interverbebral discs was uneven. Swollen furuncled dead bones scattered in them and the articular surfaces were destructed irregularly.

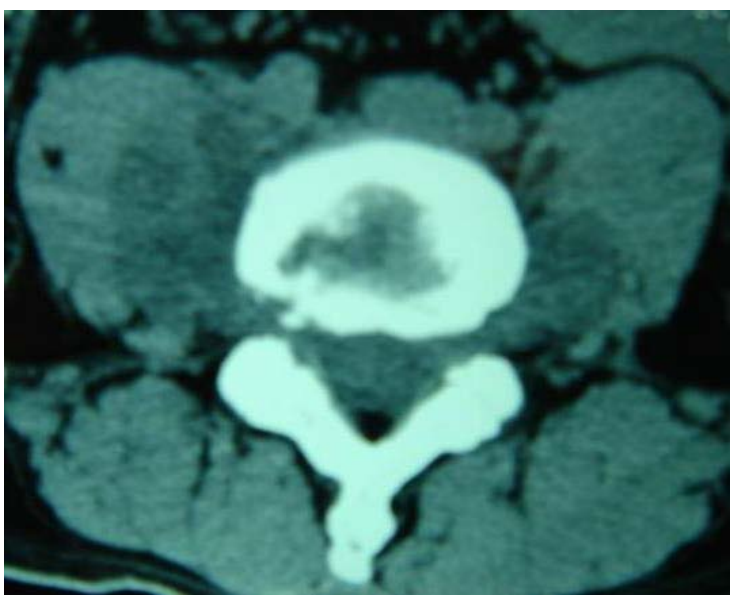

Figure 10. Male, 46 years old, Brucellar spondylitis of the L-4. Manifestation of CT: The psoas major muscles in both sides become wide in the plane where the vertebral body was destructed and in which the abscess emerged but not clear and the circumambient fat space is clear.

groups, the early detection of bone and surrounding soft tissue signal abnormalities involving the intervertebral space narrowing, vertebral body was uneven signal and found that spinal epidural abscess, or inflammatory destruction of intervertebral disc granulation tissue broke into the spinal canal, the corresponding plane spinal cord, dural sac or cauda equina compression, compression material Brucella spondylitis mostly granuloma (Figure 14), and tuberculosis of the spine, mostly disc tissue necrosis and sequestrum (Figure 15). Akman et al. [27] considered abscess edge enhancement on MRI signal prompts spinal tuberculosis. However, the present study showed

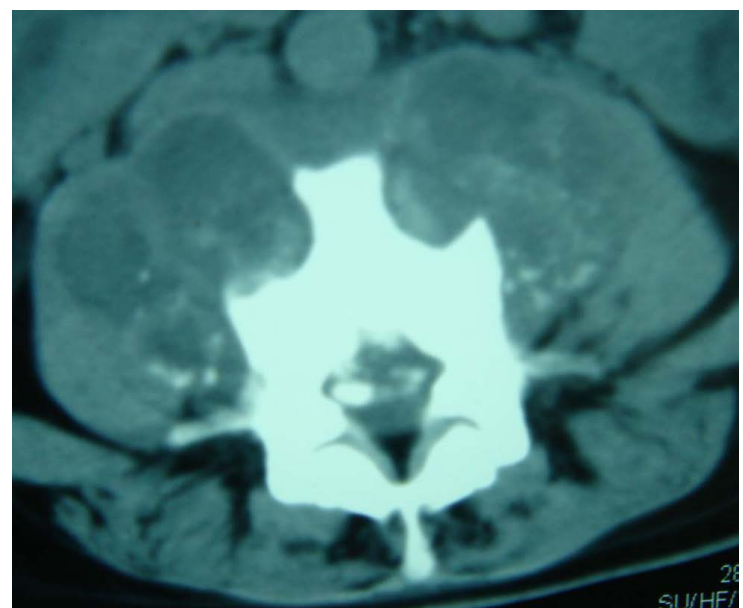

Figure 11. Female, 29 years old, the tuberculosis of the spine of the L-1. Manifestation of CT: The shadow of the abscess of psoas major muscles was large and clear in which the calcification focuses were found and the abscess was larger than the vertebral body multiple-cavity disgorged abscess formed.

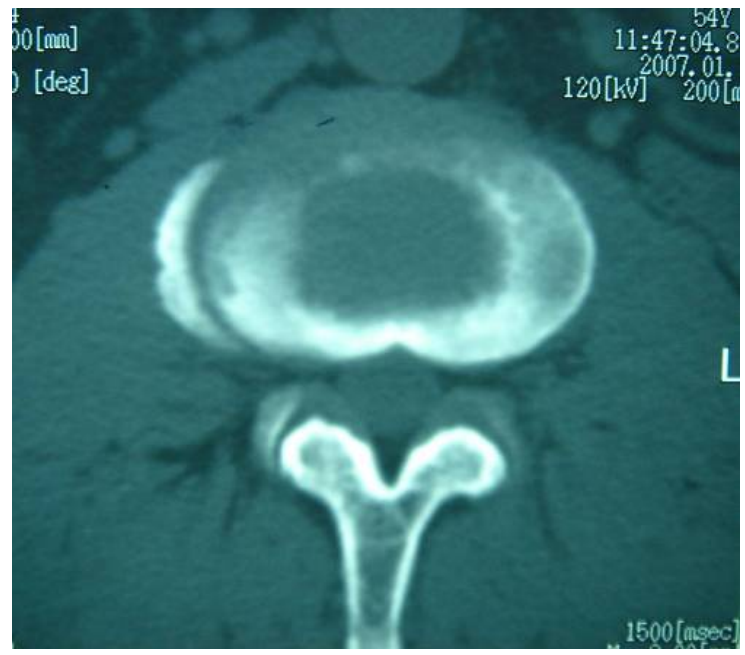

Figure 12. Male, 38 years old, Brucellar spondylitis of the L-4. Manifestation of CT: the periosteum around the vertebral bodies got hyperplastic, pachyntic and calcific and formed osteophymas like lips.

that the two groups has an edge enhancement signal abscess, but Brucella spondylitis rare epidural abscess, wherein there is no significant local tissue reaction (Figure 16), while the opposite spinal tuberculosis, etc. This point Pina [23] reported the same. Thoracolumbar spinal tuberculosis involving the vertebral body and more for osteolytic destruction, destruction of lesion or most often involving a vertebral destruction sequestrum see multiple irregularities in the kitchen, often involving the destruction of the vertebral pedicle rear, adjacent vertebral density generally reduced, local tissue reactions were; intervertebral disc damage, uneven density, there are "stars" like dead bone residue scattered in which the adjacent 


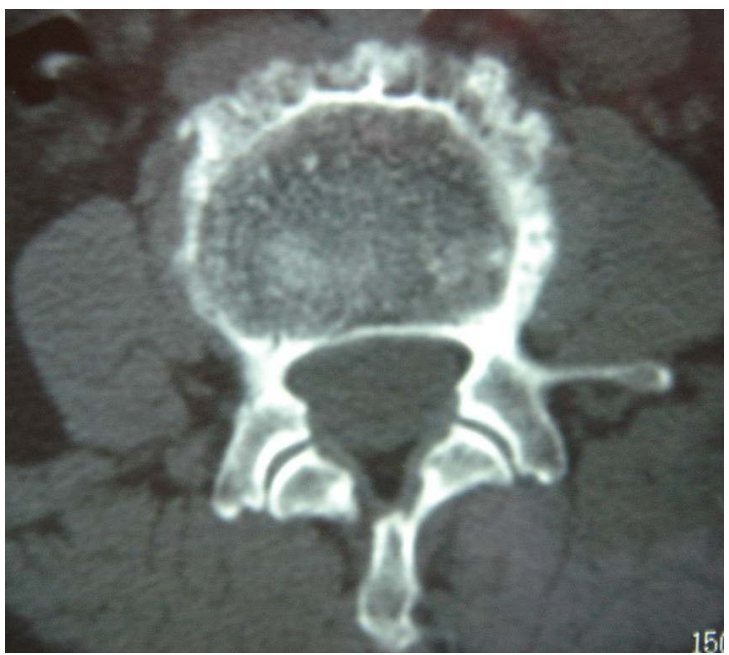

Figure 13. Male, 41 years old, Brucellar spondylitis of the L-4. Manifestation of CT: The neoformative osteophymas combined with the destruced focuses among them constituted the distinctive manifestation called lacy vertebral body.
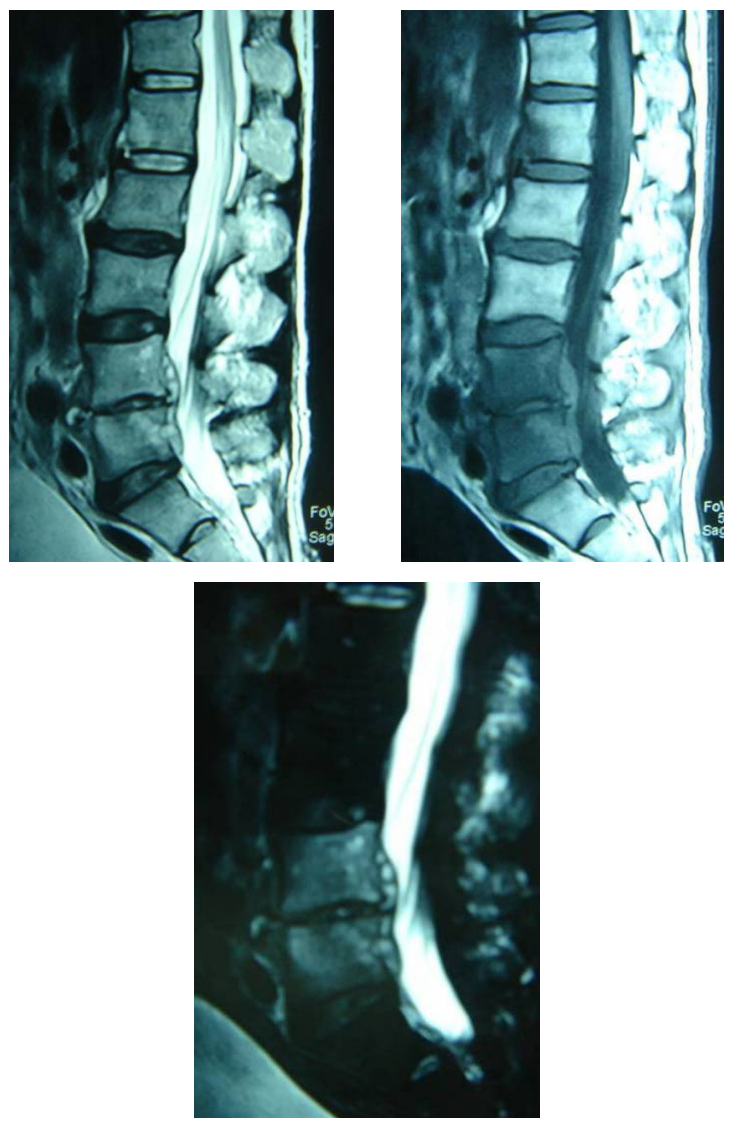

Figure 14. Male, 40 years old, Brucellar spondylitis of the L-4 and L-5. Manifestation of MRI: The pathological vertebral bodies and the intervertebral space were invaded by inflammatory matters. There was ill-defined soft tissue signal before the vertebral bodies and the spinal dura mater in the canalis vertebralis was compressed by the inflammatory granuloma.

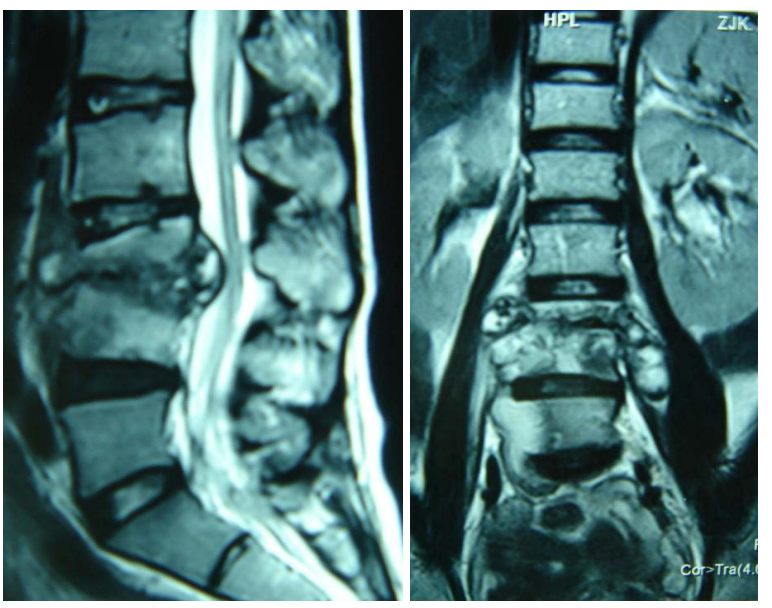

Figure 15. Male, 34 years old, the tuberculosis of the spine of the L-3 and L-4. Manifestation of MRI: Collapse of the pathological vertebral bodies, the kyphosis, destruction of the interverbebral discs, compression of the spinal dura mater by the necrotic intervertebral discs and dead bones, thin and smooth intensive abscess paries before or beside the vertebral bodies.
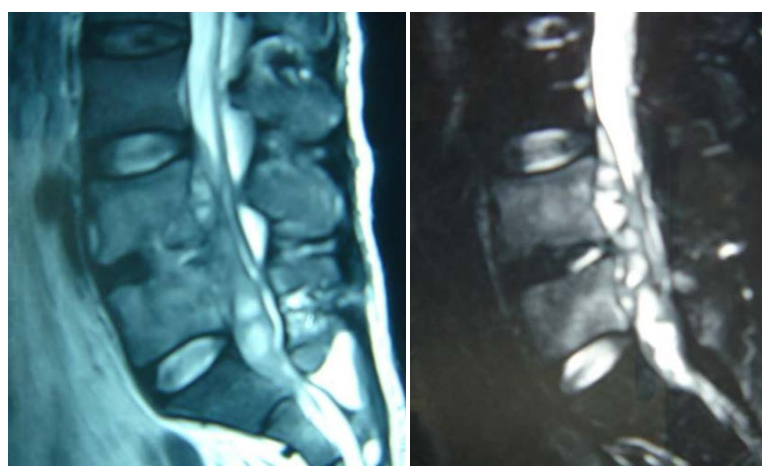

Figure 16. Male, 44 years old, Brucellar spondylitis of the L-4 and L-5. Manifestation of MRI: The epidural abscess outside the canalis vertebralis formed and the characteristic was that there was no conspicuous local tissue reaction.

vertebral endplates irregular bone destruction or disappearance, most accompanied by paraspinal abscess formation, and calcification within the common abscess, abscess often exceed the length of the vertebral lesion formation flow injection abscess, abscess edge signal enhancement [24,25]; tuberculosis of the spine is the most reliable MRI features "jump lesion" and thin and smooth abscess wall and enhanced well-defined paraspinal abnormal signal (Figure 17) [26,27]. The Brucella spondylitis and more involved in the lower lumbar vertebral segments and 1 - 2 [2,17], which is characterized by thick, irregular enhanced MRI abscess wall and ill-defined paraspinal abnormal signal, T1WI showed low signal, T2WI high signal to bone destruction evident when, T2WI high signal on fat suppression such as vertebrae, discs, accessories and intraspinal showed heterogeneous high signal (Figure 18) [2,3,28-31]. 


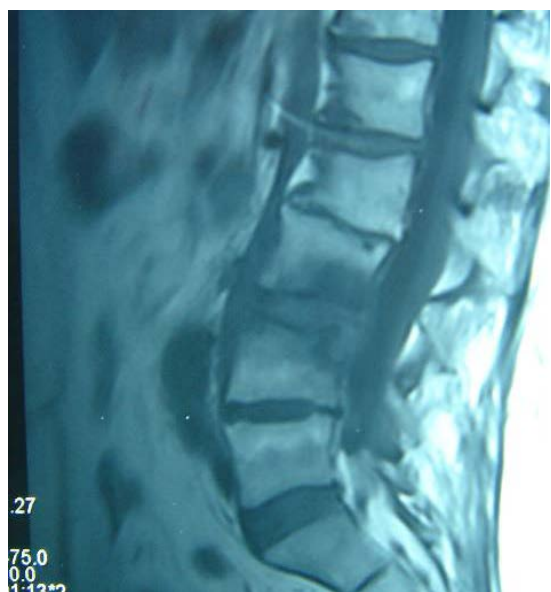

Figure 17. Male, 47 years old, the tuberculosis of the spine of the L-3 and L-4. Manifestation of MRI: The focuses were skipped and the vertebral bodies and the interverbebral discs were destructed. The thin and smooth intensive abscess paries and the abnormal well-circumscribed signal beside the vertebrae can be found.
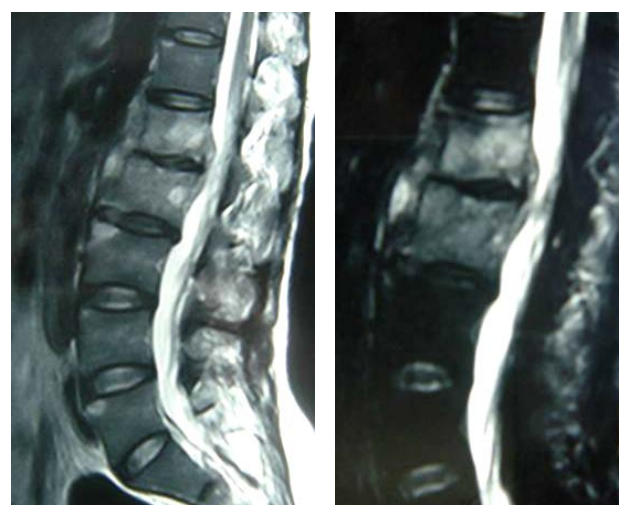

(a)
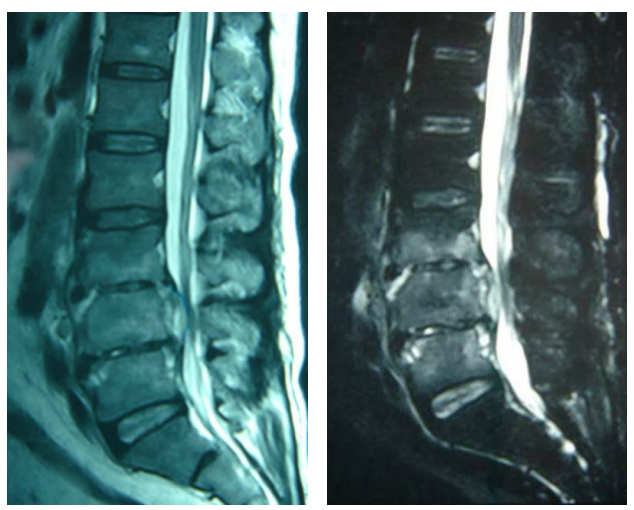

(b)

Figure 18. (a) Male, 43 years old, Brucellar spondylitis of the L-4 and L-5. (b) Male, 45 years old, Brucellar spondylitis of from L-3 to L-5. Manifestation of MRI: the thin and irregular intensive abscess paries and the abnormal ill-defined signal at beside the vertebrae-T1WI is low signal and T2WI is high signal. The vertebral bodies, intervertebral discs, accompaniment and the intra of vertebral canal are uneven high signal in the fat-suppressed sequence.

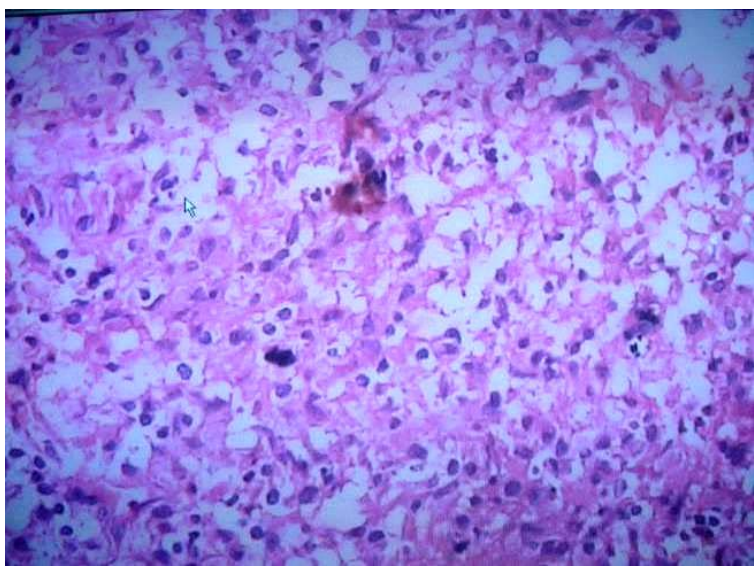

(a)

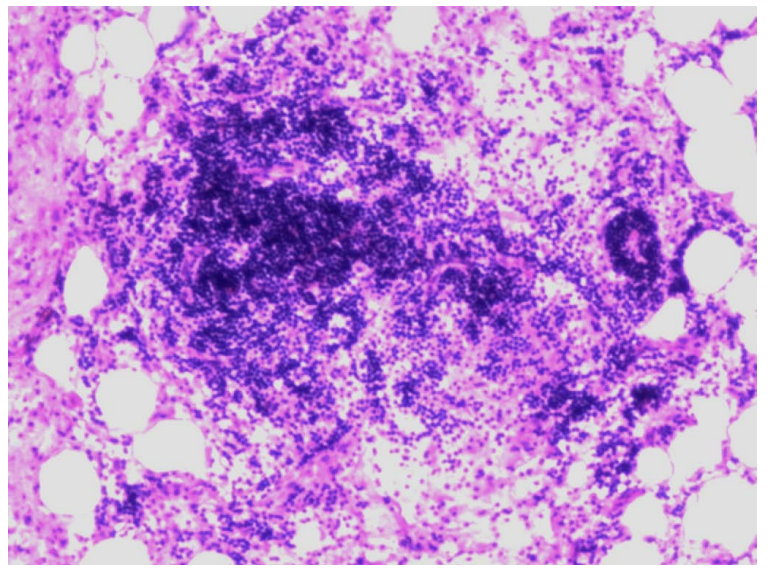

(b)

Figure 19. (a) Brucellar spondylitis. By HE staining and the microscope of $10 \times 20$ multiple hyperplasy of the histiocytes in the focuses and the formation of the proliferating nodus and uncheesy granuloma can be seen in which there are a lot of histoleucocytes, homeocytes, neutrophile granulocytes, acidophilic cells and nodular focuses made of flaky epithelioid cells without dead bone tissues. (b) By HE staining and the microscope of $10 \times 20$ multiple hyperplastic lymph tissues that have formed folliculus lymphaticus can be seen.

\subsection{Pathology}

The group of 257 cases of brucellosis spondylitis pathological examination showed the lesion tissue cell proliferation, proliferation of non-caseous nodules and granuloma formation, within which there are a large number of monocytes, lymphocytes, neutrophils, eosinophils cells infiltrated into the film composed of epithelioid cells nodular lesions, no sequestrum organizations, and the proliferation of lymphoid tissue, formation of lymphoid follicles (Figure 19). The group of 332 cases of spinal tuberculosis tuberculosis lesions reasonable examination showed nodules, nodule central caseous necrosis surrounded by epithelioid cells, Langhans giant cells and the outer periphery of the infiltrating lymphocytes, plasma 


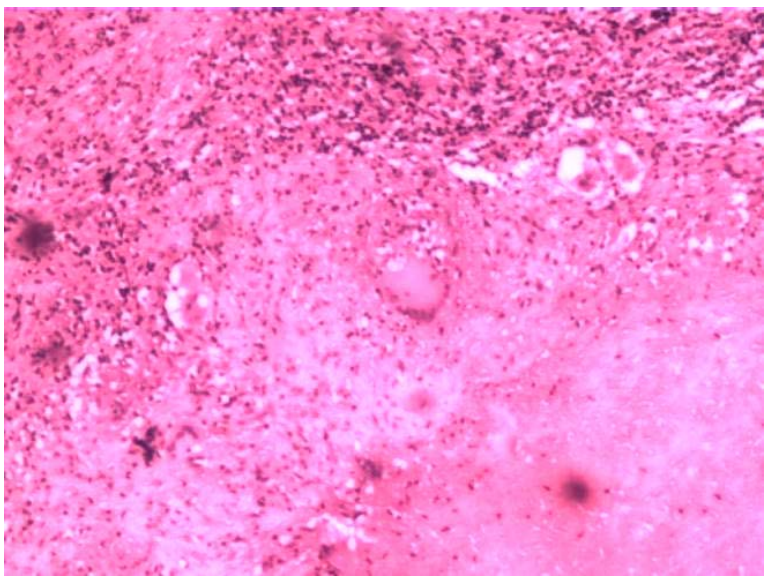

(a)

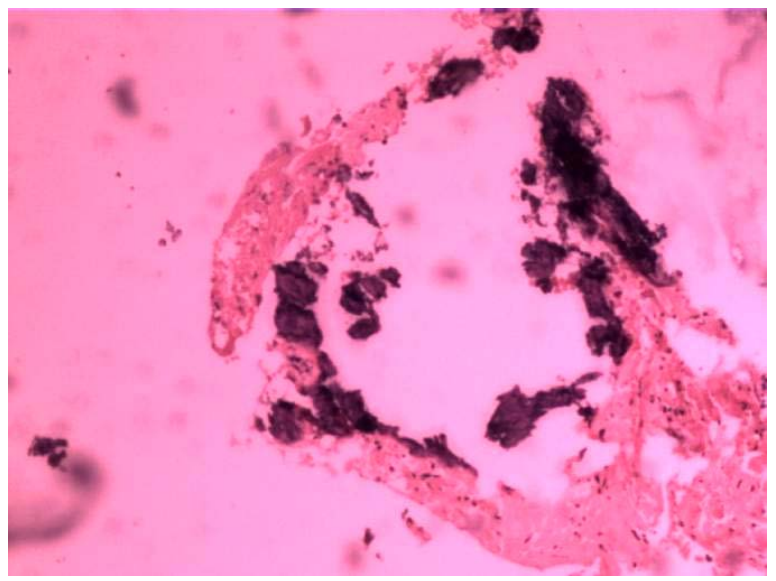

(b)

Figure 20. The tuberculosis of the spine. (a) By HE staining and the microscope of $10 \times 4$ multiple the even, red-stained and structureless tuberclers can be found and there was cheesy necrosis in the centre of the nodus and the epithelioid cells, Langhans giant cells, lymphocytes and plasma cells stellatedly spread in the peripheral areas. (b) The basophilous and trachychromatic chips of the dead bones and the degenerative fibrous connective tissues can be seen at the margin of the tubercles.

cells and a small amount of proliferation of fibroblasts, There sequestrum organization tubercle edge visible sequestrum basophils stained tissue and degeneration of fibrous connective tissue (Figure 20).

\section{REFERENCES}

[1] T. Turunc, Y. Z. Demiroglu, H. Uncu, S. Colakoglu and H. Arslan, "A Comparative Analysis of Tuberculous, Brucellar and Pyogenic Spontaneous Spondylodiscitis Patients," Journal of Infection, Vol. 55, No. 2, 2007, pp. 158-163. http://dx.doi.org/10.1016/j.jinf.2007.04.002

[2] M. H. Yilmaz, B. Mete, F. Kantarci, R. Ozaras, H. Ozer, A. Mert, I. Mihmanli, R. Ozturk and K. Kanbergoglu, “Tuberculous, Brucellar and Pyogenic Spondylitis: Comparison of Magnetic Resonance Imaging Findings and
Assessment of Its Value,” South Medical Journal, Vol. 100, No. 6, 2007, pp. 613-614. http://dx.doi.org/10.1097/SMJ.0b013e3180600eaa

[3] E. T. Tali, "Spinal Infections," European Journal of Radiology, Vol. 50, No. 2, 2004, pp. 120-133. http://dx.doi.org/10.1016/j.ejrad.2003.10.022

[4] G. Bianco, M. E. Pompeo, C. Mastroianni, G. Trasimeni, A. Paris, S. Coletta, V. Vullo, P. Serra and M. Venditti, "Non-Tubercular and Non-Brucellar Spondylodiscitis: Preliminary Clinico-Microbiologic Analysis of 37 Cases," Recenti Progressi in Medicina, Vol. 94, No. 12, 2003, pp. 554-559.

[5] A. Stabler and M. F. Reiser, "Imaging of Spinal Infection," Radiologic Clinics of North America, Vol. 39, No. 1, 2001, pp. 115-135.

[6] J. M. C. Romero, J. L. R. Salado and F. G. de la Llana, "Differences between Tuberculous Spondylitis and Brucellar Spondylitis,” Anales de Medicina Interna, Vol. 18, No. 6, 2001, pp. 309-311.

[7] X.-M. Yang, W. Shi and Y.-K. Du, "Investigation on the Curative of Brucellar Spondylitis," Chinese Journal of Epidemiology, Vol. 27, No. 6, 2008, pp. 699-703.

[8] X.-M. Yang, W. Shi and Y.-K. Du, “The Clinical Characteristics and Surgical Treatment of Brucellar Spondylitis,” Chinese Journal of Orthopedics, Vol. 28, No. 1, 2008, pp. 35-40.

[9] J. S. G. del. Pozo, M. V. Soto, M. Lizan-Garcia, E. Martinez-Alfaro, J. C. Segura-Luque and J. Solera-Santos, "Incidence of Infectious Spondylitis in the Province of Albacete (Spain)," Enfermedades Infecciosasy Microbiología Clínica, Vol. 23, No. 9, 2005, pp. 545-550.

[10] Y. A. Gokhale, A. G. Ambardekar, A. Bhasin, M. Patil, A. Tillu and J. Kamath, "Brucella Spondylitis and Sacroiliitis in the General Population in Mumbai,” Journal of the Association of Physicians of India, Vol. 51N, 2003, pp. 659-666.

[11] G. S. Guven, B. Cakir, G. Oz, M. D. Tanriover, E. Turkmen and T. Sozen, "Could Remembering the Prozone Phenomenon Shorten Our Diagnostic Journey in Brucellosis?” Rheumatology International, Vol. 26, No. 10, 2006, pp. 933-935.

http://dx.doi.org/10.1007/s00296-006-0118-3

[12] M. Turgut, A. T. Turgut and U. Kosar, "Spinal Brucellosis: Turkish Experience Based on 452 Cases Published during the Last Century,” Acta Neurochirgica, Vol. 148, No. 10, 2006, pp. 1033-1044. http://dx.doi.org/10.1007/s00701-006-0877-3

[13] G. Aydin, A. Tosun, I. Keles, E. Ayaslioglu, O. Tosun and S. Orkun, "Brucellar Spondylodiscitis: A Case Report,” International Journal of Clinical Practice, Vol. 60, No. 11, 2006, pp. 1502-1505. http://dx.doi.org/10.1111/j.1742-1241.2005.00656.x

[14] X.-M. Yang, W. Shi and Y.-K. Du, “Image Manifestations and Surgical Treatment of Spondylitis Caused by Brucells Infection,” Chinese Journal of Zoonoses, Vol. 23, No. 10, 2007, pp. 1055-1058.

[15] B. S. Tur, N. Suldur, S. Ataman, E. A. Ozturk, A. Bingol and M. B. Atay, "Brucellar Spondylitis: A Rare Cause of 
Spinal Cord Compression,” Spinal Cord, Vol. 42, No. 5, 2004, pp. 321-324.

http://dx.doi.org/10.1038/sj.sc.3101571

[16] J. S. G. del Pozo, M. V. Soto and J. Solera, "Vertebral Osteomyelitis: Long-Term Disability Assessment and Prognostic Factors,” Journal of Infection, Vol. 54, No. 2, 2007, pp. 129-134. http://dx.doi.org/10.1016/j.jinf.2006.01.013

[17] K. Z. Yuksel, M. Senoglu, M. Yuksel and M. Gul, "Brucellar Spondylo-Discitis with Rapidly Progressive Spinal Epidural Abscess Presenting with Sciatica,” Spinal Cord, Vol. 44, No. 12, 2006, pp. 805-808. http://dx.doi.org/10.1038/sj.sc.3101938

[18] P. Atonis, M. Tzermiadianos, A. Gikas, P. Papagelopoulos and A. Hadjipavlou, "Surgical Treatment of Spinal Brucellosis," Clinical Orthopaedics \& Related Research, Vol. 444, 2006, pp. 66-72. http://dx.doi.org/10.1097/01.blo.0000203455.59393.9a

[19] C. Marrakchi, B. Kilani, F. Kanoun, R. Abdelmalek, H. Tiouiri, A. Goubontini, F. Zouiten, K. Ezzaouia, M. Kooli, M. Khalfaoui and T. Ben-Chaabane, "Melitococcal Psoas Abscesses: Study of Three Cases and Review of the Literature,” Médecine Tropicale, Vol. 66, No. 3, 2006, pp. 261-265.

[20] C. B. Taarit, S. Turki and H. B. Maiz, "Infectious Spondylitis: Study of a Series of 151 Cases," Acta Orthopaedica Belgica, Vol. 68, No. 4, 2002, pp. 381-387.

[21] D. Ozol, A. Koktener and M. E. Uyar, “Active Pulmonary Tuberculosis with Vertebra and Rib Involvement: Case Report,” South Medical Journal, Vol. 99, No. 2, 2006, pp. 171-173. http://dx.doi.org/10.1097/01.smj.0000198642.28149.94

[22] M. Bosilkovski, L. Krteva, S. Caparoska and M. Dimzova, "Osteoarticular Involvement in Brucellosis: Study of 196 Cases in the Republic of Macedonia," Croatian Medical Journal, Vol. 45, No. 6, 2004, pp. 727-733.

[23] M. A. Pina, P. J. Modrego and J. J. Uro, "Brucellar Spinal
Epidural Abscess of Cervical Location: Report of Four Cases,” European Neurology, Vol. 45, No. 4, 2001, p. 249. http://dx.doi.org/10.1159/000052137

[24] M. Namiduru, I. Karaoglan and S. Gursoy, "Brucellosis of the Spine: Evaluation of the Clinical, Laboratory, and Radiological Findings of 14 Patients," Rheumatology International, Vol. 24, No. 3, 2004, pp. 125-129. http://dx.doi.org/10.1007/s00296-003-0339-7

[25] Y. Capar, S. Cesur, O. Yuksel, H. Kurt and T. H. Sozen, “Case Report: Psoas Abscess Due to Brucellosis,” Mikrobiyoloji Bülteni, Vol. 36, No. 2, 2002, pp. 219-221.

[26] D. Olivares, V. N. Lopez, R. Serrano and F. L. Garcia, "Brucellosis Complicated by a Psoas Muscle Abscess," Enfermedades Infecciosasy Microbiología Clínica, Vol. 22, No. 3, 2004, p. 200. http://dx.doi.org/10.1157/13058033

[27] S. Akman, M. Sirvanci and U. Talu, "Magnetic Resonance Imaging of Tuberculous Spondylitis,” Orthopedics, Vol. 26, No. 3, 2003, p. 69.

[28] H. P. Ledermann, M. E. Schweitzer and W. B. Morrison, "MR Imaging Findings in Spinal Infections: Rules or Myths?” Radiology, Vol. 228, No. 2, 2003, p. 506. http://dx.doi.org/10.1148/radiol.2282020752

[29] S. Moorthy and N. K. Prabhu, "Spectrum of MR Imaging Findings in Spinal Tuberculosis," American Journal of Roentgenology, Vol. 179, No. 4, 2002, pp. 979-983. http://dx.doi.org/10.2214/ajr.179.4.1790979

[30] I. Demirci, "Brucella Diskitis Mimicking Herniation without Spondylitis: MRI Findings,” Zentralbl Neurochir, Vol. 64, No. 4, 2003, pp. 178-181. http://dx.doi.org/10.1055/s-2003-44622

[31] A. Cascio, C. Iaria, A. Campenni, A. Blandino and S. Baldari, "Use of Sulesomab in the Diagnosis of Brucellar Spondylitis," Clinical Microbiology and Infection, Vol. 10, No. 11, 2004, pp. 178-181. http://dx.doi.org/10.1111/j.1469-0691.2004.00961.x 